\title{
Limited evidence to support the use of physical therapy for temporomandibular disorder
}

\author{
How effective are physical therapy interventions in the management of \\ temporomandibular disorder?
}

\section{Medlicott MS, Harris SR.}

A systematic review of the effectiveness of exercise, manual therapy, electrotherapy, relaxation training, and biofeedback in the management of temporomandibular disorder. Phys Ther 2006; 86:955-973

Data sources Medline, CINAHL (Cumulative Index to Nursing and Allied Health Literature) and Cochrane Central Register of Controlled Trials were searched, using the search terms "facial pain", "physical therapy", "rehabilitation", "temporomandibular disorder" (TMD), "temporomandibular joint" (TMJ), "temporomandibular joint syndrome" and "therapy". The search was restricted to English-language publications from 1966 to January 2005.

Study selection For inclusion, studies had to meet the following criteria: subjects were from one of three groups identified in the first axis of the Research Diagnostic Criteria for TMD; the intervention was within the realm of physical therapist practice; an experimental design was used; and outcome measures assessed one or more of the primary presenting symptoms.

Data extraction and synthesis Studies were evaluated using Sackett's rules of evidence and 10 scientific rigour criteria. One reviewer performed the literature search, study selection and data abstraction. Four randomly selected articles were also rated independently by two reviewers to assess the reliability of the first author. Effect size was also calculated for studies for which raw data were available.

Results The search identified 108 articles, of which 30 studies met the inclusion criteria. Inter-reviewer agreement was $100 \%$ for levels of evidence and $73.5 \%$ for methodological rigour. Of the 30 studies reviewed, 22 were randomised controlled trials (RCT) but of low study quality. The following recommendations arising from the 30 studies were:

- active exercises and manual mobilisations may be effective;

- postural training may be used in combination with other interventions, as independent effects of postural training are unknown;

- mid-laser therapy may be more effective than other electrotherapy modalities;

- programmes involving relaxation techniques and biofeedback, electromyography training and proprioceptive re-education may be more effective than placebo treatment or occlusal splints;

- combinations of active exercises, manual therapy, postural correction and relaxation techniques may be effective.

Conclusions These recommendations should be considered with caution because none were supported by numerous, decisive studies. Consensus on the definition of TMD, and subsequent inclusion and exclusion criteria, would allow further comparison across groups studied. In addition, agreement on use of valid and reliable outcome measures would yield more rigorous research.

Address for correspondence: MS Medlicott, 2759 Webster Rd, Nanaimo, British Columbia, Canada, V9R 6W7.E-mail: mmedlicott@hotmail.com

\section{Commentary}

The authors present an excellent overview of evidence supporting or refuting the use of various therapeutic interventions in the management of TMD. It is important to mention, however, that TMD, orofacial pain (OFP) and cervical spine disorders (CSD) are inherently related and must be considered and managed in a comprehensive, individualised approach.

The retrospective analysis used rigorous selection criteria and resulted in the inclusion of a minimal number of studies. These were primarily related to single interventional modalities, with only one representative of a comprehensive approach. This results in an emphasis upon pain relief without addressing aetiological factors, functional restoration and prevention of recurrence. ${ }^{1}$

As well as the need to improve the methodology of the RCT, the objective variables require standardisation and the reporting of all relevant parameters is paramount for replication. ${ }^{1}$ A major factor behind the deficiency in quality RCT dealing with TMD treatment by physical therapists is that only a small fraction of these practitioners specialises in the evaluation and treatment of TMD, OFP and associated CSD. Education about how physical therapy can be applied in this area is minimal and has only recently been added to the curriculum: this means that there is currently insufficient knowledge and experience practice which would need to be improved by postgraduate education.

Evidence of the efficacy of treatments is critical, but the reader must understand that a single intervention should not be used alone to manage TMD and OFP with their myriad aetiological factors, often including CSD. ${ }^{2,3}$ Individualised treatment protocols designed to restore function, decrease pain and prevent recurrence cannot be achieved by the repetitive administration of passive modalities in a dental or physical therapy office. Some of the modalities cited, however, are ideally components of a patient's home programme of exercise, postural re-education and nonmedicinal pain control. ${ }^{1,3,4}$

The physical therapy profession is now focused on research to demonstrate that a combination of manual techniques, therapeutic exercise and postural education, geared to the patient's presentation, is superior to single-modality interventions. . $^{5,6}$ Recent evidence highlights the role of posture and associated pain referral that can mimic TMD or exist as a co-morbidity: ${ }^{7,8}$ reports of this may be found in CSD publications and are included in the physical therapist's curriculum. ${ }^{9-13}$ Specialised certification also now exists for this profession in the evaluation and comprehensive management of TMD, OFP and CSD. ${ }^{14}$

\section{Jeffrey S. Mannheimer}

Department of Rehabilitation Medicine. College of Physicians and Surgeons, Columbia University, New York, USA 
1. Mannheimer JS. Nonmedicinal and noninvasive pain control techniques in the management of rheumatic disease and related musculoskeletal disorders. J Rheumatol 1987; 14 (suppl. 15):S26-S32.

2. de Wijer A, Steenks A, de Leeuw MH, Bosman F, Helders PJ. Symptoms of the cervical spine in temporomandibular disorders. J Oral Rehabil 1996; 23:742-750.

3. Kraus S. Temporomandibular disorders, head and orofacial pain: cervical spine considerations. Dent Clin N Am 2007; 51:161-193.

4. Mannheimer JS, Kraus S, Hesse J, et al. Cervicogenic mechanisms of orofacial pain and headaches. In Guidelines for the Management of Orofacial Pain and Temporomandibular Disorders. Edited by de Leeuw R. Quintessence; 2008 (in press).

5. Ismail F, Demling A, Hessling K, Fink M, Stiesch-Scholz M. Short-term efficacy of physical therapy compared to splint therapy in treatment of arthrogenous TMD. J Oral Rehabil 2007; 34:807-813.

6. Oh DW, Kim KS, Lee GW. The effect of physiotherapy on post-temporomandibular joint surgery patients. J Oral Rehabil 2002; 29: 441-446.

7. Fernandez-de-las-Penas C, Cuadrado ML, Pareja JA. Myofascial trigger points, neck mobility and forward head posture in unilateral migraine. Cephalgia 2006; 26:1061-1070.

8. Fernandez-de-las-Penas C, Alonso-Blanco C, Cuadrado ML, Gerwin RD,Pareja JA. Trigger points in the sub-occipital muscles and forward head posture in tension-type headache. Headache 2006; 46:454-460.
9. Jull G, Trott P, Potter H, et al. A randomized controlled trial of exercise and manipulative therapy for cervicogenic headache. Spine 2002; 27:1835-1843.

10. Torelli P, Jensen R, Olesen J. Physiotherapy for tension-type headache: a controlled study. Cephalgia 2004; 24:29-36.

11. McDonnell MK, Sahrmann SA, Van Dillen L. A specific exercise program and modification of postural alignment for treatment of cervicogenic headache. J Ortho Sports Phys Ther 2005; 35:3-15.

12. Stewart MJ, Maher CG, Refshauge KM, Herbert RD, Bogduk N, Nicholas M. Randomized controlled trial of exercise for chronic whiplash-associated disorders. Pain 2007; 128:59-68.

13. Van Ettekoven $\mathrm{H}$, Lucas $\mathrm{C}$. Efficacy of physiotherapy including craniocervical training program for tension-type headache; a randomized clinical trial. Cephalgia 2006; 26:983-991.

14. Mannheimer JS. The physical therapy board of craniofacial and cervical therapeutics. J Orofac Pain 2007; 21:336.

Evidence-Based Dentistry (2007) 8, 110-111. doi:10.1038/sj.ebd.6400528

\section{Key to evidence graphic used in the Evidence-based Dentistry Journal}

The graphic is based on the Centre for Evidence-based Medicine levels of Evidence tables www.cebm.net/levels_of_evidence.asp (see Evidence-based Dentistry 2003;4: p 17-18)

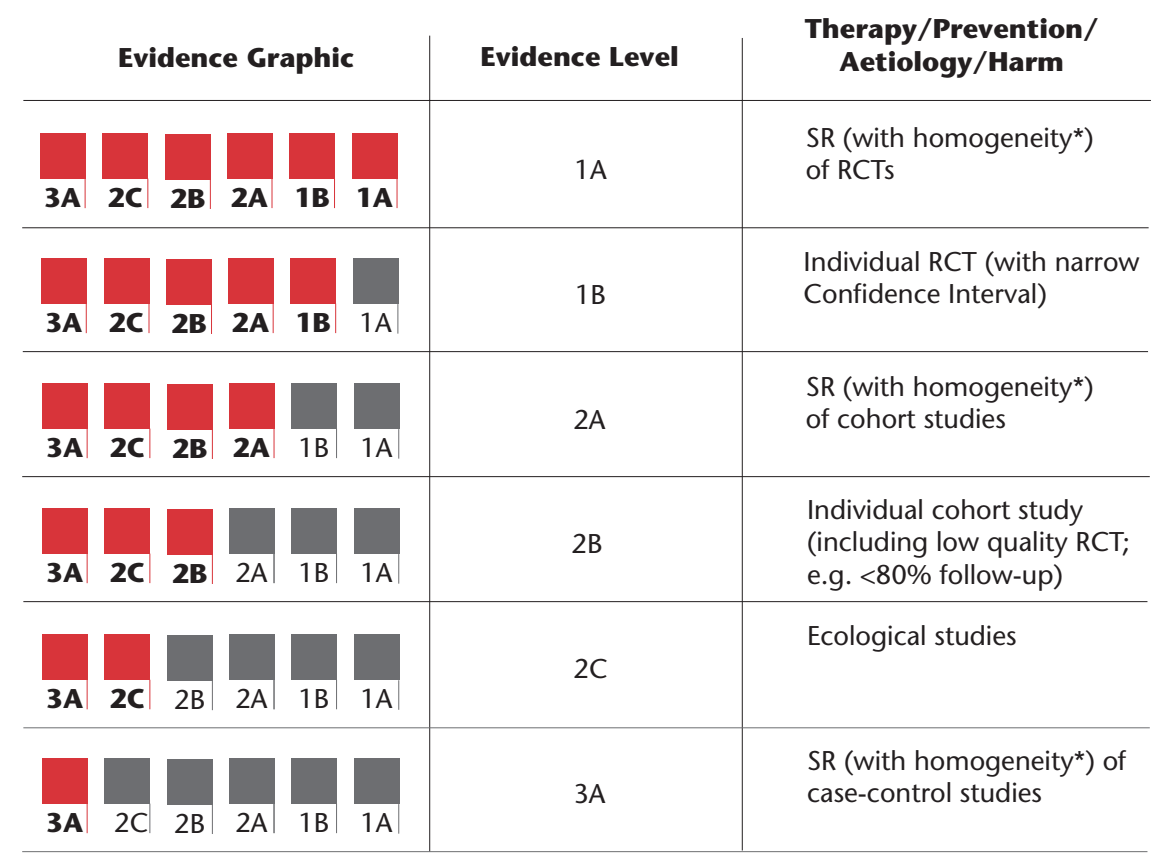

* By homogeneity we mean a systematic review that is free of worrisome variations (heterogeneity) in the directions and degrees of results between individual studies. Not all systematic reviews with statistically significant heterogeneity need be worrisome, and not all worrisome heterogeneity need be statistically significant. 